\title{
EFECTO DEL PROCESO DE FERMENTACIÓN CON BACTERIAS ÁCIDO LÁCTICAS SOBRE PROPIEDADES REOLÓGICAS DE MASAS DE MAÍZ OPM
}

\section{EFFECT OF THE FERMENTATION PROCESS WITH LACTIC ACID BACTERIA ON RHEOLOGICAL PROPERTIES OF OPM DOUGH}

\author{
Sandra Betancourt ${ }^{1}$, Alfredo Ayala ${ }^{2}$, Cristina Ramírez ${ }^{3}$
}

\begin{abstract}
${ }^{1}$ Doctora Ing. Alimentos, Universidad del Valle, Escuela de Ingeniería de Alimentos, Facultad de Ingeniería, Universidad del Valle, calle 13 \# 100-00, Cali, Valle-Colombia, e-mail: sandra.patricia.betancourt@correounivalle.edu.co; ${ }^{2}$ Doctor Ciencia y Tecnología de Alimentos, Profesor Asociado, Escuela de Ingeniería de Alimentos, Facultad de Ingeniería, Universidad del Valle, calle 13 \# 100-00, Cali, Valle-Colombia, e-mail: alfredo.ayala@correounivalle.edu.co; ${ }^{3}$ Doctora en Ingeniería de Bioprocesos, Profesora Asociada, Escuela de Ingeniería de Alimentos, Universidad del Valle, calle 13 \# 100-00, Cali, ValleColombia, e-mail: cristina.ramirez@correounivalle.edu.co
\end{abstract}

Rev. U.D.C.A Act.\& Div. Cient. 17(2): 503-511, Julio-Diciembre, 2014

\section{RESUMEN}

En Colombia, se producen, en forma tradicional y de manera empírica, masas de maíz fermentadas. El desconocimiento, tanto del proceso como de las características requeridas en el producto final y la falta de control en la fabricación, hace que las masas obtenidas no sean de calidad constante; por lo tanto, se realizó el análisis de los cambios en las propiedades reológicas de las masas, con diferentes temperaturas y tiempos de fermentación, empleando, para tal fin, un inóculo puro de Lactobacillus plantarum (CPQBA 087-11 DRM) aislado, de masas tradicionales del Valle del Cauca. Para el análisis estadístico, se aplicó un diseño experimental compuesto central rotatorio, con valores de temperatura de fermentación, con valor máximo y mínimo, de 45 y $35^{\circ} \mathrm{C}$, respectivamente y tiempo de fermentación, con valor mínimo de $12 \mathrm{~h}$ y máximo de $48 \mathrm{~h}$. Los resultados mostraron que el módulo viscoso y el ángulo de fase de las muestras, indicaron incrementos desde $1,18 \times 10^{5} \mathrm{~Pa}$ hasta valores mayores a $1,4 \times 10^{5} \mathrm{~Pa}$ y desde $11,9^{\circ}$ hasta ángulos mayores a los $20^{\circ}$, respectivamente. Por el contrario, se evidenció una disminución en el módulo de almacenamiento, con valores desde $5,59 \times 10^{5}$ hasta valores inferiores a $3 \times 10^{5} \mathrm{~Pa}$, debido a la presencia de ácidos orgánicos y enzimas amilolíticas producidas por las bacterias lácticas, las cuales, afectan la estructura de los gránulos de almidón.

Palabras clave: Masas, fermentación, bacterias, reológicas, módulos, viscoelásticas.

\section{SUMMARY}

Fermented corn doughs are produced in Colombia in a traditional and empirical way. The unawareness of both the process and the characteristics required in the final product, as well as the lack of control in the manufacturing make the obtained dough inconsistent. Therefore, analysis of changes were made to the rheological properties of doughs, using different fermentation times and temperatures, using a pure inoculum of Lactobacillus plantarum (087-11 CPQBA DRM) isolated from traditional doughs of the Valle del Cauca for this purpose. A rotatable central composite experimental design was used for statistical analysis with temperature fermentation with maximum and minimum values of 45 and $35^{\circ} \mathrm{C}$ respectively and fermentation time with minimum $12 \mathrm{~h}$ and maximum $48 \mathrm{~h}$. The results showed that the viscous modulus and the phase angle of the samples, showed increases from $1.18 \times 10^{5} \mathrm{~Pa}$ to values greater than $1,4 \times 10^{5} \mathrm{~Pa}$ and from $11.9^{\circ}$ to angles greater than $20^{\circ}$ respectively. On the contrary, a decrease in the storage modulus was noted with values from $5.59 \times 10^{5} \mathrm{~Pa}$ to values below $3 \times 10^{5} \mathrm{~Pa}$, due to the presence of organic acids and amylolytic enzymes produced by the lactic acid bacteria, which affect the structure of the starch granules.

Key words: Dough, fermentation, bacteria, rheological, moduli, viscoelastic. 


\section{INTRODUCCIÓN}

Las empanadas con masa de maíz, los tamales vallunos y las tortillas de maíz para preparar sopa son alimentos comúnmente consumidos en Colombia y son producidos, tradicionalmente, utilizando masas fermentadas. En Colombia, las áreas sembradas de maíz, para el 2008 y 2009, fueron de 623.025 y 591.890 ha, respectivamente, lo que demuestra la importancia de este cultivo (FAO, 2010). Además, la Presidencia de la República implementó el proyecto PAÍS MAÍZ, para incrementar la siembra de la variedad QPM, mediante el uso de semillas certificadas de híbridos y de transgénicos.

Las bacterias ácido lácticas -BAL-, se encuentran en una gran variedad de ambientes, incluyendo, productos lácteos, vegetales y plantas, cereales, carnes y productos cárnicos. Muchas especies de estas bacterias, se emplean para la manufactura y la conservación de víveres; en algunos alimentos, las BAL se encuentran como contaminantes o adicionadas como cultivos iniciadores, para controlar los procesos de fermentación (Mayo et al. 2010). Las masas tradicionales colombianas, se fabrican mediante el remojo de los granos de maíz crudo, por un período entre tres y siete días, cambiando el agua diariamente, exponiendo los tanques de preparación al sol durante el día y resguardándolos durante la noche, sin la utilización de cultivos iniciadores para el proceso.

El contenido de una flora natural de bacterias ácido lácticas en cereales se ha destinado, por tradición, en una gran variedad de productos, algunos de ellos son el kenkey y el mawè, que se fabrican comúnmente en África, a base de maíz. Se ha demostrado que la fermentación ácido láctica tiene un impacto favorable, no sólo en el sabor, el aroma y la textura, sino en el aumento en la digestibilidad de este cereal (Nout, 2009).

Este tipo de masas también podrían ser aprovechadas para la producción de panes libres de gluten, ya que se ha presentado un aumento en el número de personas con enfermedad celíaca (Green \& Cellier, 2007) y las opciones de panes en el mercado, a base de maíz y arroz, son de baja calidad, tanto en su sabor como en su textura (Niewinski, 2008). Debido a que las masas deben ser lo suficientemente elásticas y viscosas para facilitar su procesamiento y su aplicación en productos de panificación, se requiere del estudio de sus propiedades viscoelásticas, mediante el análisis reológico dinámico, como los componentes elástico y viscoso, los cuales, pueden ser afectados por el proceso de fermentación con BAL amilolíticas.

Los métodos reológicos miden las propiedades viscoelásticas macroscópicas de la masa y sus componentes, donde, $\mathrm{G}^{\prime}$ es conocido como el módulo de almacenamiento, que determina el comportamiento elástico, mientras que el mó- dulo G"', se conoce como módulo de pérdida y describe el comportamiento viscoso del material analizado. El componente elástico $G^{\prime}$ es una medida de la energía guardada en el material en deformación sinusoidal y recuperada por ciclo, mientras que el componente viscoso G' ' es la medida de la energía disipada o pérdida en forma de calor por ciclo de deformación (Mirsaeedghazi et al. 2008). Finalmente, el ángulo de fase, se calcula mediante la relación entre $G^{\prime \prime}$ y $G^{\prime}$ y es la medida de la contribución relativa de cada componente (Masi et al. 2001).

El objetivo de esta investigación fue analizar el comportamiento de las propiedades reológicas de una masa de maíz sometida a tratamientos de tiempo y de temperatura de fermentación, mediante el uso de la cepa Lactobacillus plantarum (CPQBA 087-11 DRM) aislada de masas ácidas, tradicionalmente producidas en el Valle del Cauca, Colombia y caracterizada como amilolítica.

\section{MATERIALES Y MÉTODOS}

Microorganismo: Durante el proceso de fermentación, se empleó la bacteria Lactobacillus plantarum (CPQBA 087-11 DRM), como cultivo iniciador, que fue aislado de masas ácidas, tradicionalmente producidas en diferentes municipios del Valle del Cauca, Colombia. A este microorganismo, se le realizó, por anticipado, la caracterización, mediante pruebas de producción de ga,s a partir de glucosa, resistencia a $\mathrm{pH}$ bajo, determinación de la actividad amilolítica, análisis de producción de ácidos orgánicos por cromatografía líquida de alta eficiencia (HPLC), detección de los isómeros del ácido láctico, poder de inhibición frente a bacterias patógenas y actividad antifúngica in vitro, ensayos de competitividad, pruebas de actividad proteolítica, pruebas bioquímicas e identificación molecular, para asegurar que las cepas fueran seguras, para su uso en alimentos (Betancourt et al. 2013).

Producción de harina y masas de maíz: Para la producción de la harina y la masa de maíz, se manejó maíz QPM, donado por el Consorcio Latinoamericano y del Caribe de Apoyo a la Investigación y al Desarrollo de la Yuca, CLAYUCA; para la obtención de la masa, los granos crudos y secos fueron molidos y tamizados con malla Tyler número 30 . La harina se empacó en bolsas plásticas al vacío y se almacenó a $8^{\circ} \mathrm{C}$, en una nevera comercial.

El inóculo, se preparó en medio de cultivo MRS (De Man et al. 1960), hasta el tiempo de máxima obtención de células. Debido a que el medio de cultivo utilizado no es apto para el consumo humano, la suspensión de células obtenida se centrifugó a 5000rpm, durante 10min; se separó el sobrenadante y se le adicionó agua estéril. A partir de esta concentración de células, se tomó una alícuota de la suspensión y se realizó la inoculación de un medio líquido a base de maíz $(5 \mathrm{~g}$ de 
harina de maíz QPM/100mL de agua); posteriormente, la harina se inoculó con la suspensión de células, obteniendo una masa con el $10 \%$ de inóculo, con una relación harina:agua de 1,0:0,8. La inoculación e hidratación, se realizaron en cabina de flujo laminar y, la homogenización, se llevó a cabo en una mezcladora de laboratorio limpia y desinfectada, de marca Walita Máster, Brasil. Igualmente, se usaron guantes estériles, para asegurar asepsia en la manipulación de las muestras. Para establecer la homogeneidad de la mezcla, se efectuaron pruebas de conteo de células viables a diferentes tiempos de mezclado, en puntos aleatorios de la masa.

Las masas fueron prensadas en recipientes plásticos sellados, para asegurar anaerobiosis y, la temperatura de fermentación, se controló utilizando incubadoras programadas a las temperaturas obtenidas, a partir del diseño experimental.

Diseño experimental: Para el análisis estadístico, se aplicó el diseño experimental central compuesto con dos factores, que son temperatura de fermentación, con valor máximo y mínimo de 45 y $35^{\circ} \mathrm{C}$, respectivamente y, tiempo de fermentación, con valor mínimo de $12 \mathrm{~h}$ y máximo de $48 \mathrm{~h}$; el diseño experimental cuenta con cinco niveles ( 2 factorial, 2 axial, 1 central). El diseño experimental, se ajustó a un modelo de segundo orden (Ecuación 1):

$$
y=\beta_{o}+\sum_{i=1}^{k} \beta_{i} x_{i}+\sum_{i=1}^{k} \beta_{i i} x_{i}^{2}+\sum_{i<j} \sum \beta_{i j} x_{i} x_{j}+\varepsilon
$$

Las variables de respuesta fueron los módulos G', G' ' y el ángulo de fase. Los valores experimentales fueron analizados utilizando el programa Minitab $14 \AA$.

Medición de pH: A cada muestra fermentada, se le determinó el pH, aprovechando un equipo Thermo Scientific (Orion 3 Star, Singapur); para ello, se tomaron $10 \mathrm{~g}$ de masa y se le adicionaron $100 \mathrm{~mL}$ de agua destilada. La muestra se mantuvo en agitación durante $10 \mathrm{~min}$ y se procedió a realizar la medición.

Determinación de propiedades viscoelásticas: A cada muestra, correspondiente a los diferentes tiempos y temperaturas de fermentación, se le determinaron los parámetros viscoelásticos G', G' ' y el ángulo de fase, mediante pruebas dinámicas de oscilación, empleando un reómetro rotacional (Bohlin Rotational Rheometer CVO 100D, UK). Se utilizó un sistema de platos paralelos de $20 \mathrm{~mm}$ de diámetro y distancia (Gap), entre platos de $2 \mathrm{~mm}$. Previo a la medición, todas las muestras se dejaron en reposo durante 15min, para permitir la relajación de las mismas (Magaña-Barajas et al. 2009). Cada muestra, se colocó en la placa del reómetro y el exceso del material se retiró con una espátula plástica. El borde expuesto de la muestra, se cubrió con una fina capa de aceite de parafina ligero, para prevenir la evaporación durante las mediciones.
Se aplicó un test de barrido de esfuerzo, para identificar la región viscoelástica lineal. Con base en este resultado, se determinó un nivel de esfuerzo dentro de esta región lineal; posteriormente, a todas las muestras fermentadas y no fermentadas, se le realizaron un barrido de frecuencias, entre $0,1 \mathrm{y} 10 \mathrm{~Hz}$. Todas las pruebas se efectuaron a $25^{\circ} \mathrm{C}$.

\section{RESULTADOS Y DISCUSIÓN}

Comportamiento de $\mathrm{pH}$ en función de la temperatura y tiempo de fermentación: El valor inicial del $\mathrm{pH}$ para la masa sin tratamiento con BAL fue de 6,11 $\pm 0,2$ y al ser inoculada con BAL, alcanzó un pH de 5,65 $\pm 0,1$. Este resultado es esperado, ya que la suspensión de BAL presenta producción de ácidos orgánicos durante la fermentación (Reddy et al. 2008).

La figura 1 muestra el comportamiento del $\mathrm{pH}$ en función de la temperatura y el tiempo de fermentación. Se observa, que las muestras en todos los tratamientos mostraron valores de $\mathrm{pH}$ inferiores a 4,0, para tiempos superiores a $25 \mathrm{~h}$, en los intervalos de temperatura entre 33 y $47^{\circ} \mathrm{C}$, del proceso de fermentación, que se encuentra en el rango mesófilo, apto para el crecimiento de las BAL.

Valores bajos en el pH $(4,05)$ fueron obtenidos por Valmorri et al. (2008), durante la fermentación de masas ácidas fermentadas típicas italianas, utilizando S. cerevisiae/L. plantarum en cocultivo. De igual modo, Plessas et al. (2008) realizaron fermentaciones de masas de trigo, con Kluyveromyces marxianus, Lactobacillus delbrueckii ssp. bulgaricus y Lactobacillus helveticus, a 30 y $40^{\circ} \mathrm{C}$, alcanzando un valor de $\mathrm{pH}$ de 4,3 , para temperaturas cercanas a $40^{\circ} \mathrm{C}$. Katina et al. (2007) obtuvieron 3,7 de pH durante la fermentación de centeno, con adición de $L$. plantarum, a $30^{\circ} \mathrm{C}$, durante 20h. Igualmente, De Angelis et al. (2006) fermentaron masas de centeno con una mezcla probiótica comercial, a $37^{\circ} \mathrm{C}$, durante $24 \mathrm{~h}$, alcanzando valores de $\mathrm{pH}$ de 3,8 y Moore et al. (2007) aprovechando dos cepas de L. plantarum (LP 2115 KW y LP FST 1.11), para la fermentación de harina de trigo durante $48 \mathrm{~h}$, obteniendo valores de $\mathrm{pH}$ de 3,87 y 3,81, respectivamente.

Propiedades reológicas: Para la realización de las pruebas reológicas dinámicas, se determinó previamente la zona viscoelástica lineal, mediante un barrido de esfuerzo, el cual se ejecutó, con la finalidad de asegurar que no se presentan cambios estructurales, debido a la aplicación de esfuerzos elevados durante las pruebas de barrido de frecuencia. Como resultado, se seleccionó un esfuerzo de $15 \mathrm{~Pa}$.

En la figura 2, se muestra la superficie de respuesta para el módulo de almacenamiento $\mathrm{G}^{\prime}$, que presenta un valor mí- 


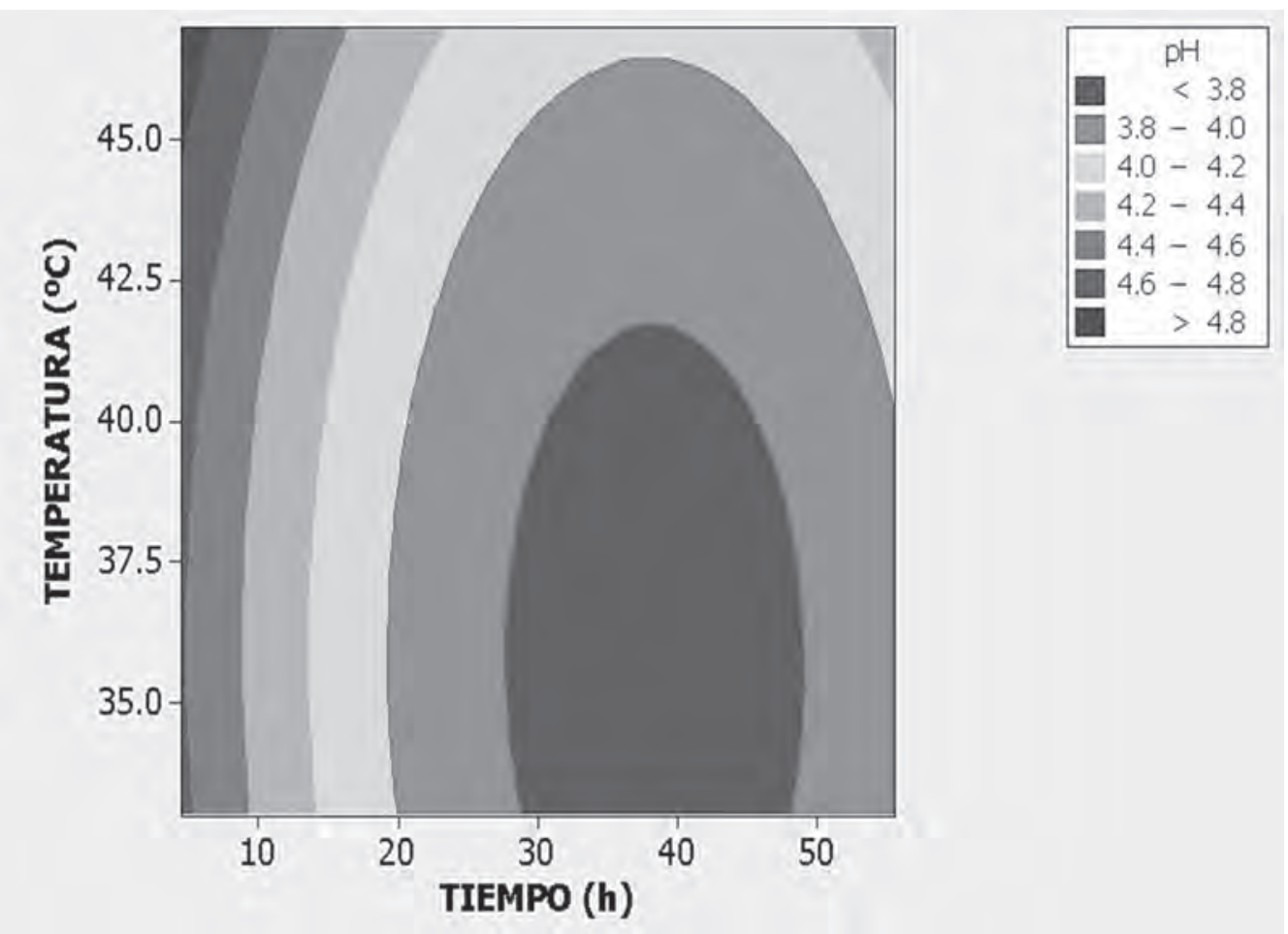

Figura 1. Comportamiento del pH en función de la temperatura y tiempo de fermentación.

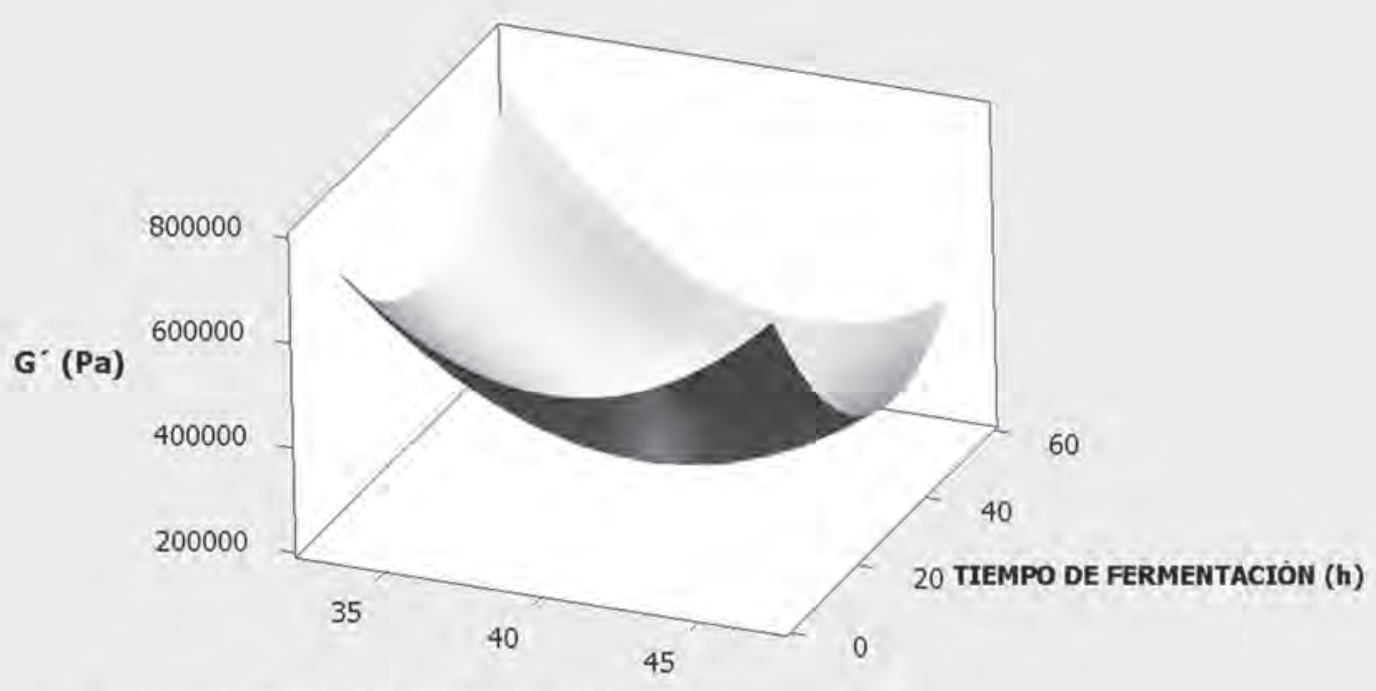

TEMPERATURA DE FERMENTACIÓN ( $\left.{ }^{\circ} \mathrm{C}\right)$

Figura 2. Superficie de respuesta para el módulo de almacenamiento (G'). 
nimo, para referencias cercanas a $40^{\circ} \mathrm{C}$ y $30 \mathrm{~h}$ de fermentación. Estos valores bajos de $\mathrm{G}^{\prime}$ muestran una reducción en el dominio de sus propiedades elásticas, que está relacionada directamente con los totales mínimos de $\mathrm{pH}$ obtenidos, durante el proceso de fermentación. Este comportamiento puede ser atribuido a que durante los procesos de fermentación con BAL, se presenta la hidrólisis ácida y enzimática de los gránulos de almidón. Esta hidrólisis, se reportó en el estudio de Haydersah et al. (2012), donde se mostró un cambio en la consistencia de los productos de mezclas espesas a base de almidón, hacia productos de consistencia más líquida; de igual manera, se evidenció la producción de maltooligosacáridos en los productos amiláceos fermentados.

Para un nivel de significancia del $5 \%$, se tiene que, en este modelo, la constante, los parámetros asociados a la temperatura, la temperatura al cuadrado, el tiempo al cuadrado y la relación tiempo-temperatura resultaron estadísticamente significativos $(\mathrm{p}<0.05)$, para el modelo ajustado; es así que el modelo de segundo orden ajustado está dado por la ecuación 2:

$$
\hat{y}=6810145-313141 x_{1}+3964 x_{1}^{2}+308 x_{2}^{2}-418 x_{1} x_{2}
$$

El coeficiente de determinación $\mathrm{R}^{2}$, para este modelo, fue 0,9461 , lo que significa que el $94,61 \%$ de la variabilidad del módulo de almacenamiento $\left(\mathrm{G}^{\prime}\right)$, se explica por el modelo de superficie de respuesta ajustado.
La figura 3 muestra la superficie de respuesta para el módulo viscoso. El ANOVA evidenció un efecto significativo $(p<0.05)$ de la temperatura y tiempo de fermentación sobre G". Se nota que, para valores de temperatura de fermentación entre 35 y $42^{\circ} \mathrm{C}$ y tiempos de fermentación alrededor de las $30 \mathrm{~h}$, se obtienen los mayores valores del módulo G' '. Estos valores de G' ' demuestran que, para los valores más bajos de $\mathrm{pH}$ obtenidos, por la fermentación con BAL, se logran masas con mayor suavidad, es decir, que tienen una mayor tendencia a comportarse como un material líquido, debido a la acción de las enzimas amilolíticas y ácido orgánicos, que provocan la hidrólisis de los gránulos de almidón, lo que facilita los procesos de amasado y mezclado, durante la producción de alimentos a base de maíz.

La ecuación 3 muestra el modelo conseguido para la variable $G^{\prime \prime}$, con un nivel de significancia de $5 \%(p<0,05)$; además, se obtuvo un $\mathrm{R}^{2}$ de $84,6 \%$ y se cumplió el supuesto de normalidad de los residuos, con un valor de la media cercana a cero, de $5,7372 \times 10^{-14}$.

$\hat{y}=-613173+40223 x_{1}-526 x_{1}^{2}$

El ángulo de fase para los procesos de fermentación a diferentes tiempos y temperaturas, se muestran en la figura 4, donde se observa que la superficie de respuesta presenta forma cóncava hacia abajo, con un ángulo de fase con un valor máximo de $24,5^{\circ}$ y $\tan (\delta)=0,45$, aproximadamente a

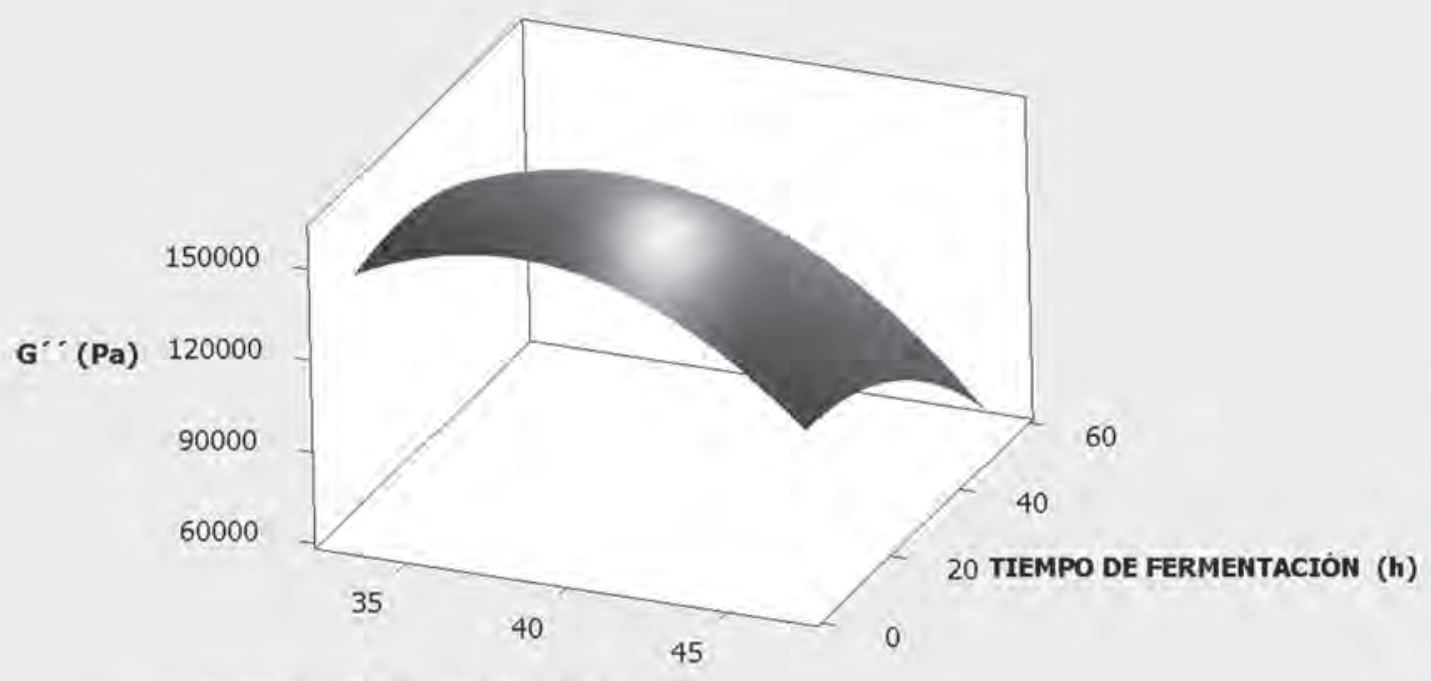

TEMPERATURA DE FERMENTACION ( $\left.{ }^{\circ} \mathrm{C}\right)$

Figura 3. Superficie de respuesta para el módulo viscoso (G' $)$. 


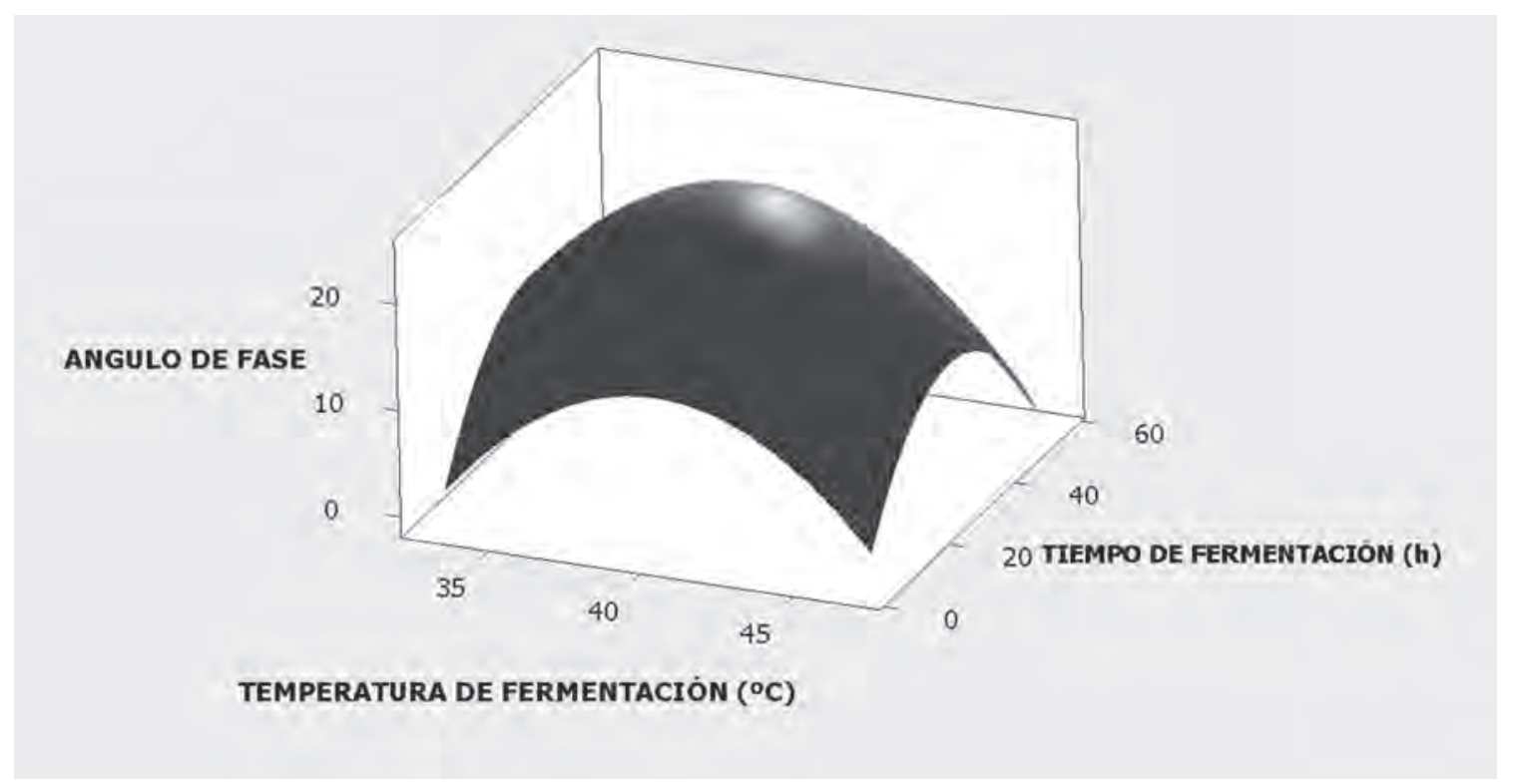

Figura 4. Superficie de respuesta para el ángulo de fase.

$40^{\circ} \mathrm{C}$ y $30 \mathrm{~h}$ de fermentación. Los valores obtenidos para la $\tan (\delta)$ en todos los tratamientos, se encuentran entre 0,2 y 0,6 , lo que indica que las muestras se comportan como un gel débil (Juszczak et al. 2012). Este comportamiento, se detectó también en masas libres de gluten a base de almidones modificados de papa y maíz con alta amilasa, durante el estudio de Witczak et al. (2012).

El aumento del ángulo de fase demuestra que las masas fermentadas sufren un mayor cambio estructural para las condiciones de proceso, donde los valores de $\mathrm{pH}$ son menores. Clarke et al. (2002) notaron que la adición de masas ácidas fermentadas producidas utilizando cultivos iniciadores de BAL, a masas de panificación, presentaba un incremento en el ángulo de fase comparado con la masa sin adición o para masas acidificadas químicamente.

La figura 5 muestra curvas típicas del comportamiento reológico de los módulos $G^{\prime}$ y G' ' de una muestra sin fermentar y una muestra fermentada $\left(40^{\circ} \mathrm{C}, 4,5 \mathrm{~h}\right)$, durante el barrido de frecuencias a $25^{\circ} \mathrm{C}$; las otras muestras fermentadas con distintas condiciones de temperatura y de tiempo de fermentación, manifestaron similar comportamiento reológico.

Se observa que tanto la curva del módulo $G^{\prime}$ como la del módulo G' ', se incrementan durante el barrido de frecuencias, notándose mayores valores en las curvas del módulo elástico $\left(\mathrm{G}^{\prime}\right)$. Se advierte, además, que las muestras sin fermentar muestran mayores valores de $G^{\prime}$ que las muestras fermentadas, lo cual, significa que las muestras sin fermen- tar presentan dominio del carácter elástico, es decir, que su comportamiento tiende a ser como el de un material sólido (Acevedo et al. 2013).

De igual manera, se evidencian mayores valores del módulo viscoso $\left(G^{\prime \prime}\right)$ en la muestra fermentada, lo que indica un comportamiento con mayor tendencia hacia el de un líquido; de otra manera, indica que el proceso de fermentación suaviza la masa. Este aumento en el módulo G' ' de la masa fermentada puede ser atribuida a la producción de ácidos orgánicos y enzimas amilolíticas por parte de la cepa utilizada en el proceso, ya que la especie Lactobacillus es la que emplea, de manera más eficiente el almidón, como fuente de carbono y para la producción de energía (Haydersah et al. 2012). Algunas cepas que hacen parte del grupo BALA (Bacterias Ácido Lácticas Amilolíticas) son Lactobacillus amylophilus, L. amylovorus, L. acidophilus, L. fermentum y L. plantarum.

Las enzimas y los ácidos orgánicos producidos por las bacterias lácticas atacan las zonas amorfas del gránulo de almidón produciendo dextrinas y glucosa, presentándose un efecto plastificante (Reddy et al. 2008).

Este comportamiento viscoelástico de masas de maíz fue reportado durante el estudio de Quintanar et al. (2011), para barridos de frecuencia entre 0,1 y $100 \mathrm{~Hz}$. Igualmente, el predominio del carácter elástico en masas de maíz, se evidenció durante el estudio de Casarrubias-Castillo et al. (2012), para barridos de frecuencia entre $0,1 \mathrm{y} 10 \mathrm{~Hz}$, a $25^{\circ} \mathrm{C}$. 


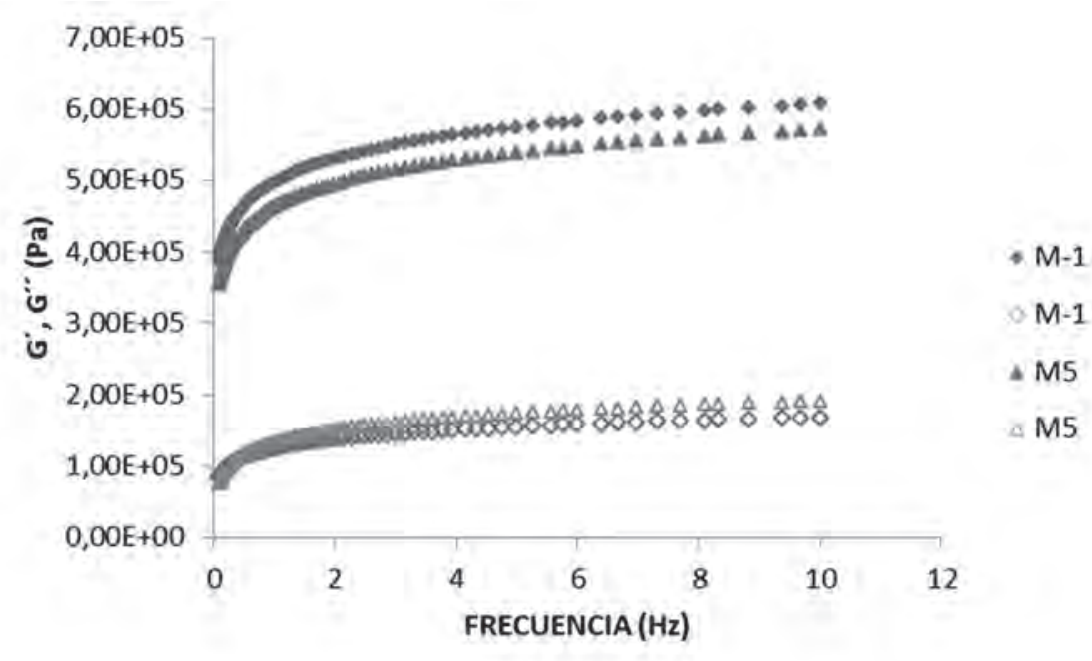

Figura 5. Barrido de frecuencias a $25^{\circ} \mathrm{C}$, para la muestra sin fermentar (M-1) y muestra fermentada (M5); los marcadores sin relleno representan $\mathrm{G}^{\prime \prime}$ y los marcadores con relleno sólido representan los valores de G'.

La figura 6 muestra el comportamiento típico del ángulo de fase para la muestra sin fermentar (M-1) y para una muestra fermentada (M5). El ángulo de fase ( $\delta$ ) mostró un incremento a medida que aumentó la frecuencia para las dos muestras, lo cual significa, un incremento relativo en el valor del módulo G' '; sin embargo, los ángulos obtenidos se encuentran en valores cercanos a $0^{\circ}$ (sólido de Hook), corroborando el predominio del comportamiento elástico ( $\left.G^{\prime}\right)$ sobre el viscoso $\left(G^{\prime \prime}\right)$. Al comparar las muestras fermentadas y no fermentadas, se evidenció que las fermentadas expusieron el mayor valor del ángulo de fase, lo cual, corrobora que el proceso de fermentación provoca una disminución de la viscosidad en las masas de maíz.

Masi et al. (2001) lograron un aumento, tanto en el módulo elástico como en el viscoso, durante el barrido de frecuencias para masas de trigo sin fermentar, mientras que los reportados por Newberry et al. (2002), no mostraron diferencias estadísticamente significativas en el módulo elástico $\left(G^{\prime}\right)$, ni en el módulo viscoso $\left(G^{\prime \prime}\right)$, entre masas con levadu-

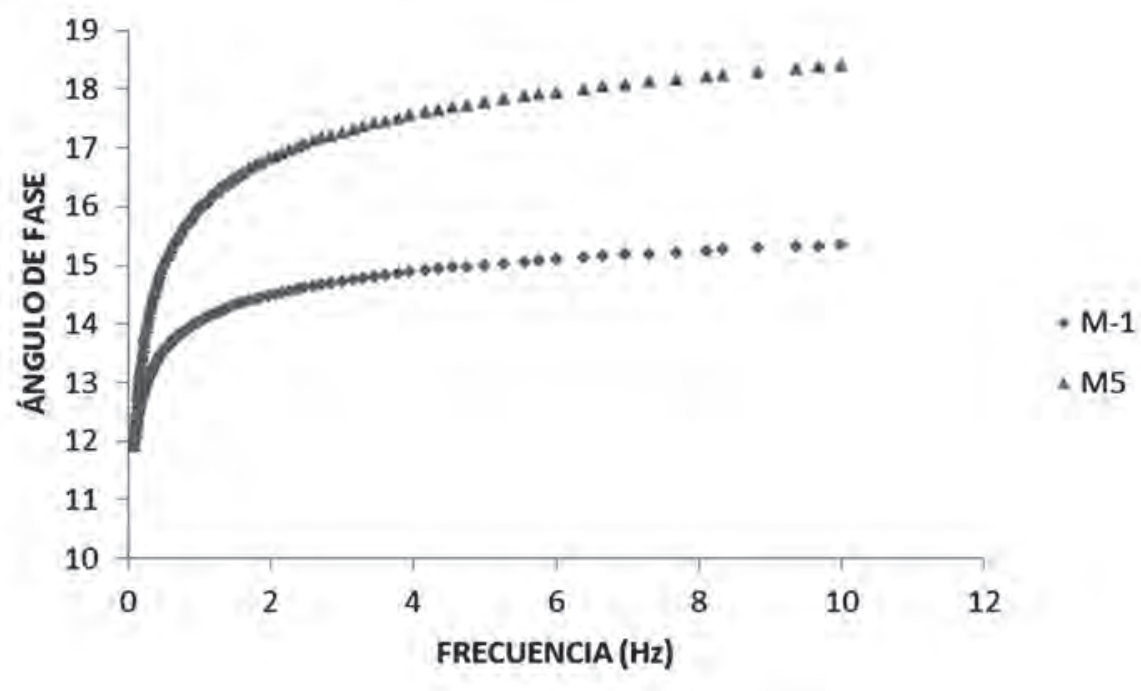

Figura 6. Comportamiento típico del ángulo de fase para muestra sin fermentar (M-1) y una de las muestras fermentadas (M5). 
ra y sin levadura, durante 120 min de fermentación. Por otra parte, Angioloni et al. (2006) reportan que a dos tiempos de fermentación (2 y 4 h), el valor de $G^{\prime}$ presenta una disminución, tanto para masa de panificación con levadura (BY) como para masa ácida fermentada (SD) activa.

Estos cambios se pueden explicar, debido a la variación en el nivel de $\mathrm{pH}$ causado por la producción de ácido láctico, lo que altera el comportamiento reológico de la masa, ya que, incluso, pequeños cambios químicos y físicos, provocan variaciones en la estructura del almidón y, en el caso de harinas a base de trigo, también se ve afectada la red de gluten, ocasionando variaciones significativas en las características reológicas de este tipo de masas (Wehrle \& Arendt, 1998). Moore et al. (2007) encontraron que las masas fermentadas de trigo con bacterias ácido lácticas fueron más suaves, debido a la producción de ácidos orgánicos, los cuales, aumentan la solubilidad de la glutenina e hidrolizan el almidón.

Con base en los resultados anteriores, se evidenció que las masas de maíz, independientemente de la presencia de Lactobacillus plantarum (CPQBA 087-11 DRM) y del proceso de fermentación, mostraron un predominio del comportamiento elástico sobre el viscoso en cada tratamiento; sin embargo, al comparar el módulo viscoso G' ' entre las muestras fermentadas y no fermentadas, se observó que las masas fermentadas indicaron mayor comportamiento viscoso, por lo que tienden a ser más suaves, por la fermentación láctica. De igual manera, se evidenció que las propiedades reológicas de las masas de maíz fermentadas están directamente relacionadas con el $\mathrm{pH}$, mostrando que, a medida que disminuyen los valores de $\mathrm{pH}$, se disminuye el comportamiento elástico, posiblemente, a los cambios estructurales en los gránulos de almidón, provocados por la hidrólisis ácida y enzimática, debida a la fermentación con la bacteria $L$. plantarum (CPQBA 087-11 DRM).

Financiación: Este estudio fue financiado por la Vicerrectoría de Investigaciones de la Universidad del Valle, a través del proyecto 2615. Conflictos de intereses: El manuscrito fue preparado y revisado con la participación de todos los autores, quienes declaramos que no existe conflicto de intereses, que ponga en riesgo la validez de los resultados presentados.

\section{BIBLIOGRAFÍA}

1. ACEVEDO, D.; MARRUGO, Y.; MONTERO, P. 2013. Evaluación de las propiedades reológicas de pastas de ajonjolí artesanal y tecnificada. Rev. U.D.C.A Act. \& Div. Cient.16(1):245-251.

2. ANGIOLONI, A.; ROMANI, S.; PINNAVAIA, G.; DALLA ROSA, M. 2006. Characteristics of bread making doughs: influence of sourdough fermentation on the fundamental rheological properties. Eur Food Res. Tech. Alemania. 222:54-57.

3. BETANCOURT, S.P.; BOLIVIAR, G.A.; RAMÍREZ, C. 2013. Fermentación de maíz de alta calidad proteica con Lactobacillus plantarum (CPQBA 087-11 DRM) aislado en Colombia de masas tradicionales fermentadas. Revista Argentina de Microbiología. 45(4):282283.

4. CASARRUBIAS-CASTILLO, M.G.; MÉNDEZ-MONTEALVO, G.; RODRÍGUEZ-AMBRIZ, S.L.; SÁNCHEZ-RIVERA, M.M.; BELLO-PÉREZ, L.A. 2012. Diferencias estructurales y reológicas entre almidones de frutas y cereales. Agrociencia. México. 46(5):455-466.

5. CLARKE, C.I.; SCHOBER, T.J.; ARENDT, E.K. 2002. Effect of single strain and traditional mixed strain starter cultures on rheological properties of wheat dough and on bread quality. Cereal Chem. USA. 79:640-647.

6. DE MAN, J.C.; ROGOSA, M.; SHARPE, M.E. 1960. A medium for the cultivation of Lactobacilli. J. Appl. Bact. 23:130-135.

7. DE ANGELIS, M.; RIZELLO, C.G.; FASANO, A.; CLEMENTE, M.G.; DE SIMONE, C.; SILANO, M.; DE VINCENZI, M.; LOSITOE, I.; GOBETTI, M. 2006. VSL\#3 probiotic preparation has the capacity to hydrolyze gliadin polypeptides responsible for Celiac Sprue probiotics and gluten intolerance. Biochimica et Biophysica Acta (BBA) - Molecular Basis of Disease. USA. 1762(1):80-93.

8. FAO. 2010. Statistical Databases (FAOSTAT). Disponible desde Internet en: http://faostat.fao.org (con acceso 12/12/2013).

9. GREEN, P.; CELLIER, C. 2007. Celiac disease. NEJM. 357(17):1731-1743.

10. HAYDERSAH, J.; CHEVALLIER, I.; ROCHETTE, I.; MOUQUET-RIVIER, C.; PICQ, C.; MARIANNE-PÉPIN, T.; ICARD-VERNIÈRE, C.; GUYOT, J.P. 2012. Fermentation by amylolytic lactic acid bacteria and consequences for starch digestibility of plantain, breadfruit, and sweet potato flours. J. Food Science. USA. 77(8):466-472.

11. JUSZCZAK, L.; WITCZAK, T.; ZIOBRO, T.; KORUS, J.; CIEŚLIK, E.; WITCZAK, M. 2012. Effect of inulin on rheological and thermal properties of gluten-free dough. Carbohydrate Polymers. USA. 90:353-360. 
12. KATINA, K.; LIUKKONEN, K.H.; KAUKOVIRTA-NORJA, A.; ADLERCREUTZ, H.; HEINONEN, S.M.; LAMPI, A.M.; PILABA, J.M.; POUTANEN, K. 2007. Fermentation-induced changes in the nutritional value of native or germinated rye. J. Cereal Science (USA). 46:348-355.

13. MAGAÑA-BARAJAS, E.; RAMÍREZ-WONG, B; PLATT-LUCERO, L.C.; LÓPEZ-AHUMADA, G.A.; TORRES, P.I.; SÁNCHEZ-MACHADO, D.I. 2009. Caracterización viscoelástica de masas de variedades de trigos suaves. Tecnol. Ciencia Ed. (IMIQ). México. 24(1):12-22.

14. MASI, P.; CAVELLA, S.; PIAZZA, L. 2001. An Interpretation of the Rheological Behavior of Wheat Flour Dough Based on Fundamental Tests En: Fergus, M. (ed) Bread Sraling. Clydesdale, Series Editor University of Massachusetts, Amherst. USA. p.75-89.

15. MAYO, B.; ALEKSANDRZAK-PIEKARCZYK, T.; FERNÁNDEZ, M.; KOWALCZYK, M.; ALVAREZ-MARTÍN, P.; BARDOWSKI, J. 2010. Updates in the Metabolism of Lactic Acid Bacteria en: Biotechnology of Lactic Acid Bacteria: Novel Applications. Wiley-Blackwell Ed. USA. p.3-33.

16. MIRSAEEDGHAZI, H.; EMAM-DJOMEH, Z.; MOUSAVI, S.M.A. 2008. Rheometric measurement of dough rheological characteristics and factors affecting it. Int. J. Agri. Biol. Pakistan. 10:112-119.

17. MOORE, M.M.; JUGA, B.; SCHOBER, T.J.; ARENDT, E.K. 2007. Effect of lactic acid bacteria on properties of gluten-free sourdoughs, batters, and quality and ultrastructure of gluten-free bread. Cereal Chem. 84(4):357-364.

18. NEWBERRY, M.P.; PHAN-THIEN, N.; LARROQUE, O.R.; TANNER, R.I.; LARSEN, N.G. 2002. Dynamic and elongation rheology of yeasted bread doughs. Cereal Chem. 79 (6):874-879.

19. NIEWINSKI, M.M. 2008. Advances in celiac disease and gluten-free diet. J. Am. Diet. Assoc. USA.108(4):661-72.
20. NOUT, M.J.R. 2009. Rich nutrition from the poorest - cereal fermentations in Africa and Asia. Food Microbiol. USA. 26:685-692

21. PLESSAS, S.; FISHER, A.; KOURETA, K.; PSARIANOS, C.; NIGAM, P.; KOUTINAS, A. 2008. Application of Kluyveromyces marxianus, Lactobacillus delbrueckii ssp. bulgaricus and L. helveticus for sourdough bread making Food Chem.106:985-990.

22. QUINTANAR, A.; JARAMILLO, M.E.; SOLORZA, J.; MÉNDEZ, M.G.; WANG, Y. 2011. Rheological and thermal properties of masa as related to changes in corn protein during nixtamalization. J. Cereal Science. 53:139-147.

23. REDDY, G.; ALTAF, M.; NAVEENA, B.J.; VENKATESHWAR, M.; VIJAY- KUMAR, E. 2008. Amylolytic bacterial lactic acid fermentation - A review. Biotechn. Adv. USA. 26:22-34.

24. VALMORRI, S.; MORTENSEN, H.D.; JESPERSEN, L.; CORSETTI, A.; GARDINI, F.; SUZZI, G.; ARNEBORG, N. 2008. Variations of internal pH in typical Italian sourdough yeasts during co-fermentation with lactobacilli. LWTdoi:10.1016/j.lwt.2007.11.006 1610-1615.

25. WEHRLE, K.; ARENDT, K. 1998. Rheological changes in wheat sourdough during controlled and spontaneous fermentation. Cereal Chem. 75(6):882-886.

26. WITCZAK, M.; JUSZCZAK, L.; ZIOBRO, T.; KORUS J. 2012. Influence of modified starches on properties of gluten-free dough and bread. Part I: Rheological and thermal properties of gluten-free dough. Food Hydrocolloids. USA. 28:353-360.

Recibido: Junio 11 de 2014

Aceptado: Octubre 13 de 2014

Como citar:

Betancourt, S.; Ayala, A.; Ramírez, C. 2014. Efecto del proceso de fermentación con bacterias ácido lácticas sobre propiedades reológicas de masas de maíz QPM. Rev. U.D.C.A Act. \& Div. Cient. 17(2): 503-511. 